\section{Modelo assistencial e indicadores de qualidade da assistência: percepção dos profissionais da atenção primária à saúde}

\author{
Health care model and quality indicators: \\ perceptions of primary health \\ care professionals
}

\author{
1 Departamento de \\ Odontologia, Universidade \\ Estadual de Montes Claros \\ Montes Claros, Brasil. \\ 2 Departamento de Saúde \\ da Mulher e da Criança, \\ Universidade Estadual \\ de Montes Claros, Montes \\ Claros, Brasil. \\ Correspondência \\ A. P. Caldeira \\ Departamento de Saúde \\ da Mulher e da Criança, \\ Universidade Estadual de \\ Montes Claros. \\ Campus Universitário \\ Professor Darcy Ribeiro, \\ Montes Claros, $M G$ \\ 39401-089, Brasil. \\ antonio.caldeira@unimontes.br
}

\begin{abstract}
This study aimed to verify the adequacy of family health teams according to the quality standards set by the Brazilian Ministry of Health and the correlation with health care activities, according to the health professionals themselves. The Evaluation for Quality Improvement (EQI) instrument was used in joint interviews with physicians, nurses, and dentists. Scores were defined for each of the dimensions, according to the number of standards met under the EQI. The lowest scores on the "consolidation of the model" dimension were for community participation and social control. For health care, the lowest scores were for adolescents' health. The sub-dimensions "reception and humanization" and "health promotion" were statistically associated with child, adult, and elderly health care. The sub-dimension "work organization" was statistically associated with child and elderly health care. A positive correlation thus exists between the model's consolidation and quality improvement.
\end{abstract}

Quality of Health Care; Health Personnel; Family Health
José Mendes da Silva 1

Antônio Prates Caldeira ${ }^{2}$
No final dos anos 1980 e início dos anos 1990, muitos países ocidentais iniciaram o processo de reforma sanitária conhecido como terceira geração das reformas do setor saúde, que na América Latina alcançou intensidade e características diferenciadas internamente e em relação aos países europeus 1 . No Brasil, o movimento de reestruturação do modelo assistencial à saúde iniciou-se após a implantação do Sistema Único de Saúde (SUS) e, sobretudo, a partir da criação do Programa Saúde da Família (PSF), em 1994. A experiência bem sucedida do Programa Agentes Comunitários de Saúde (PACS) no Nordeste do país parece ter sido um fator desencadeante desse processo, dentro de um contexto político e de incentivo financeiro aos municípios, no SUS 2.

Historicamente marcado por um modelo de assistência curativista e hospitalocêntrico, centrado na consulta médica, o sistema de saúde brasileiro carecia de mudanças profundas e radicais. Esse aspecto sofreu rupturas com o desenvolvimento da atenção primária, através de equipes multiprofissionais, estratégica e prioritariamente implantadas nas áreas de maior vulnerabilidade das cidades. Reafirmando os princípios do SUS, o PSF se expandiu rapidamente e passou a assumir o status de "estratégia" 3 . O número de equipes de saúde da família passou de pouco mais de 2 mil equipes em 1998 para aproximadamente $30 \mathrm{mil}$ equipes em 2008. A rápida expansão reafirmou 
a importância no novo modelo como prática reorganizadora dos cuidados primários de saúde. A partir de então, tornou-se necessário discutir questões relacionadas à qualificação e resolubilidade das equipes de saúde. Sobretudo, porque a maioria dos profissionais que se integram às equipes de saúde da família ainda se formam dentro de uma lógica curativista, de assistência centrada na doença e não na promoção da saúde.

Discutindo a substituição do modelo tradicional pela Estratégia Saúde da Família (ESF), Teixeira ${ }^{4}$ destaca que além da dimensão técnico-assistencial, relacionada às práticas de trabalho, outras dimensões devem ser envolvidas no processo de mudança. A autora aponta que os aspectos gerenciais ou político-institucionais e organizativos são também fundamentais no processo de mudança e afirma que a transformação do modelo exige a sinergia entre as três dimensões apresentadas.

Não há dúvidas de que, ao longo dos últimos anos a ESF tem contribuído de forma significativa para a melhoria dos indicadores de saúde no país 5,6,7. Todavia, não é possível afirmar de forma absoluta o quanto a implementação da estratégia tem implicado em efetiva reestruturação do modelo assistencial 4,8 . Tampouco é possível perceber, sem ferramentas avaliativas adequadas, os alcances e limites da estratégia. Discussões similares são necessárias para resgatar o nível de transformação do modelo assistencial que a ESF tem alcançado ou pode alcançar.

Seguramente, a expansão da estratégia propiciou a ampliação do acesso aos serviços básicos de saúde e deixou os profissionais de saúde mais próximos dos usuários 9 . Esse fato, todavia, não implica mudança dos processos de trabalho desses profissionais, que, mantendo a mesma lógica da assistência centrada na consulta médica desenvolvem parcamente os atributos da atenção primária 10

O Ministério da Saúde apresentou em 2005 uma proposta de avaliação formativa específica para a ESF, dirigida aos diversos atores envolvidos 11. Focada na auto-avaliação e apresentando padrões de referência para a organização dos serviços, a proposta de Avaliação para Melhoria da Qualidade da Estratégia Saúde da Família (AMQ) não se constitui em uma atividade final, mas uma atividade-meio, que estimula e orienta para alcance da qualidade dos serviços.

A literatura nacional apresenta poucas referências sobre o tema e as experiências de utilização dos instrumentos propostos também carecem de maior divulgação e padronização ${ }^{12}$. O instrumento tal como proposto pelo Ministério da Saúde é composto por duas unidades de análise, uma focada na gestão e outra voltada às equi- pes da ESF. A primeira aborda as subdimensões relacionadas ao desenvolvimento da ESF, coordenação técnica e estrutura das unidades de saúde, sendo dirigida aos gestores de saúde e coordenadores da atenção primária dos municípios. A outra unidade de análise representa o objeto desse estudo, aborda as dimensões de consolidação do modelo e atenção à saúde, sendo dirigida às equipes de saúde. Todos os eixos avaliativos são apresentados nos questionários do AMQ distribuídos em cinco estágios de desenvolvimento da estratégia (elementar, em desenvolvimento, consolidado, bom e avançado). Tal classificação pressupõe níveis distintos de complexidade a ser alcançada. Todavia, a definição de escores e o estabelecimento de pontos de cortes específicos para a classificação apresentada não são definidos e não possibilitam ranqueamento das equipes. Apesar de tais limitações, o instrumento tem o mérito inconteste de apresentar padrões de referência de qualidade a serem atingidos. Cultiva de tal forma, a busca da excelência, mais do que o simples inquérito avaliativo.

O instrumento proposto pelo Ministério da Saúde apresenta, conforme explicitado, duas dimensões específicas para auto-avaliação das equipes de saúde da família (consolidação do modelo de atenção e atenção à saúde). O presente estudo teve como objetivo identificar o grau de desenvolvimento das dimensões apresentadas, estabelecendo correlação entre ambas. Esse aspecto permitirá conhecer, através da percepção das equipes de saúde o quanto a consolidação do novo modelo assistencial se associa com as ações de saúde desenvolvidas por tais equipes.

\section{Métodos}

Trata-se de um estudo transversal, descritivo, realizado através de um inquérito entre os profissionais das equipes da ESF, com abordagem quantitativa. A pesquisa foi realizada no Município de Montes Claros, ao norte do Estado de Minas Gerais. Com uma população estimada superior a 360 mil habitantes, a cidade é o principal pólo urbano regional.

Informações pertinentes a este estudo são aquelas referentes à assistência dos serviços de atenção primária no município. A cidade conta com 49 equipes de saúde da família, (44 localizados na zona urbana e 5 na zona rural), o que representa uma cobertura de aproximadamente $50 \%$ para a população em relação à ESF. Existem ainda 15 equipes de agentes comunitários de saúde e 14 centros de saúde, sendo estes responsáveis pela população que não possui cobertura da ESF. 
Não houve cálculo amostral, pois o estudo faz parte de um projeto amplo que incorpora todas as equipes de saúde. A coleta de dados foi realizada com as três categorias de profissionais de nível superior (médico, enfermeiro e cirurgiãodentista) que atuam nas unidades da ESF da zona urbana do município.

Para o presente estudo a coleta de dados utilizou os formulários 4 e 5 do projeto AMQ, do Ministério da Saúde, referentes às dimensões de "consolidação do modelo de atenção" e "atenção à saúde".

A dimensão "consolidação do modelo de atenção" inclui 70 questões referentes aos seguintes temas: organização do trabalho em saúde da família; acolhimento, humanização e responsabilização; promoção da saúde; participação comunitária e controle social e vigilância à saúde.

A dimensão de "atenção à saúde" inclui 88 questões referentes aos resultados das ações sobre os distintos ciclos de vida, a saber: saúde da criança; saúde do adolescente; saúde do adulto (homens e mulheres); saúde do idoso e também informações sobre a vigilância às doenças infecto-contagiosas.

As entrevistas com os profissionais foram conduzidas nas próprias unidades de saúde em horário de trabalho. A equipe de entrevistadores era composta por duas enfermeiras e um cirurgião-dentista treinados para a coleta de dados. Os dados foram processados através do programa SPSS for Windows, versão 16.0 (SPSS Inc., Chicago, Estados Unidos). Após computados os dados, verificou-se a correlação entre os escores das subdimensões de "consolidação do modelo de atenção" com os escores das subdimensões de "atenção à saúde" através do coeficiente de correlação de Pearson. O nível de significância admitido foi de 5\%. A normalidade dos escores de cada subdimensão foi aferida através do teste de Kolmogorov-Smirnov.

Os entrevistados assinaram Termo de Consentimento Livre e Esclarecido. O presente estudo foi realizado após manifestação explícita de apoio dos gestores de saúde local e após parecer favorável do Comitê de Ética em Pesquisa da Universidade Estadual de Montes Claros.

\section{Resultados}

A coleta de dados foi concluída para 43 equipes, com a participação de 129 profissionais de nível superior: médicos, enfermeiros e cirurgiõesdentistas. Em uma das equipes alocadas não foi possível a coleta de dados para as três categorias profissionais, sendo esta excluída da análise.
Os entrevistados possuíam idade entre 26 e 46 anos, com mediana de 37 anos. A distribuição foi uniforme entre as três categorias alocadas para o estudo, com 43 médicos, 43 enfermeiros e 43 cirurgiões-dentistas. A maioria dos respondentes era do sexo feminino $(66,7 \%)$ e as características gerais das equipes revelaram que em 24 delas todos os profissionais possuíam formação específica para a ESF, sendo que os médicos concluíram a residência de medicina de família e comunidade e os enfermeiros e cirurgiões-dentistas concluíram a residência multiprofissional em saúde da família. O tempo aferido de existência das equipes nos territórios variou de um a 10,4 anos.

$\mathrm{O}$ desempenho geral das equipes da ESF, em relação às dimensões e subdimensões do AMQ, na percepção dos profissionais de saúde é apresentado na Tabela 1, que aponta a meta a ser alcançada, os valores mínimos e máximos obtidos pelas equipes (escores de conformidade). Nenhuma das equipes participantes atingiu pontuação máxima em todos os eixos de avaliação.

Os escores mais baixos da dimensão de consolidação do modelo foram referentes à participação comunitária e controle social. Para a dimensão de atenção à saúde, os escores mais baixos foram relativos à atenção à saúde do adolescente.

A Tabela 2 apresenta os resultados das correlações estabelecidas entre as subdimensões de consolidação do modelo e de atenção à saúde. As subdimensões "acolhimento, humanização e responsabilização" e "promoção da saúde" mostraram-se estatisticamente associados com a atenção à saúde da criança, do adulto e do idoso.

A subdimensão "organização do trabalho em saúde da família” mostrou-se estatisticamente associada com a atenção à saúde da criança e do idoso. Não se registrou associação das subdimensões da consolidação do modelo com atenção à saúde do adolescente.

\section{Discussão}

Registrou-se através do presente estudo uma correlação positiva entre a consolidação do modelo assistencial e as ações de atenção à saúde para a criança, para o adulto e para o idoso. A correlação observada indica que as equipes com maior adequação ao novo modelo de assistência proposto pela ESF apresentam melhores resultados nas ações de atenção à saúde, traduzidos em maiores escores nas subdimensões avaliadas. A adequação ao modelo e as ações de atenção à saúde estão bem definidas nos instrumentos do AMQ e representam padrões de qualidade a 
Tabela 1

Autopercepção de desempenho das equipes da Estratégia Saúde da Família (ESF) para as dimensões do projeto de Avaliação para Melhoria da Qualidade da Estratégia Saúde da Família (AMQ). Montes Claros, Minas Gerais, Brasil, 2008.

\begin{tabular}{|c|c|c|c|c|}
\hline \multirow[t]{2}{*}{ Dimensões/Subdimensões } & \multirow{2}{*}{$\begin{array}{l}\text { Escores alcançáveis } \\
\text { em cada subdimensão }\end{array}$} & \multicolumn{3}{|c|}{ Escores das equipes } \\
\hline & & Mínimo & Máximo & Média \\
\hline \multicolumn{5}{|l|}{ Consolidação do modelo de atenção } \\
\hline Organização do trabalho em saúde da família & 21 & 9 & 21 & 15,63 \\
\hline Acolhimento, humanização e responsabilização & 12 & 3 & 12 & 8,67 \\
\hline Promoção da saúde & 21 & 3 & 18 & 12,77 \\
\hline Participação comunitária e controle social & 4 & 0 & 4 & 1,26 \\
\hline Vigilância à saúde & 12 & 3 & 12 & 8,88 \\
\hline \multicolumn{5}{|l|}{ Atenção à saúde } \\
\hline Saúde da criança & 24 & 11 & 24 & 18,88 \\
\hline Saúde do adolescente & 6 & 0 & 5 & 2,63 \\
\hline Saúde do adulto (homens e mulheres) & 38 & 18 & 38 & 27,53 \\
\hline Saúde do idoso & 7 & 1 & 7 & 4,72 \\
\hline Vigilância às doenças infecto-contagiosas & 13 & 6 & 13 & 11,26 \\
\hline
\end{tabular}

Tabela 2

Correlação entre os escores das dimensões de consolidação do modelo de atenção e da atenção à saúde na percepção dos profissionais das equipes da Estratégia Saúde da Família (ESF). Montes Claros, Minas Gerais, Brasil, 2008.

\begin{tabular}{|c|c|c|c|c|c|c|c|c|}
\hline \multirow{4}{*}{$\begin{array}{l}\text { Dimensão de consolidação } \\
\text { do modelo de atenção }\end{array}$} & \multicolumn{8}{|c|}{ Dimensão de atenção à saúde } \\
\hline & \multirow{2}{*}{\multicolumn{2}{|c|}{ Saúde da criança }} & \multirow{2}{*}{\multicolumn{2}{|c|}{ Saúde do adolescente }} & \multirow{2}{*}{\multicolumn{2}{|c|}{$\begin{array}{l}\text { Saúde do adulto } \\
\text { (homens e mulheres) }\end{array}$}} & \multirow{2}{*}{\multicolumn{2}{|c|}{$\begin{array}{c}\text { Saúde } \\
\text { do idoso }\end{array}$}} \\
\hline & & & & & & & & \\
\hline & $r$ & $\mathrm{p}$ & $r$ & $\mathrm{p}$ & $r$ & $\mathrm{p}$ & $r$ & $\mathrm{p}$ \\
\hline Organização do trabalho em saúde da família & 0,497 & 0,001 * & 0,098 & 0,530 & 0,193 & 0,216 & 0,325 & 0,034 * \\
\hline Acolhimento, humanização e responsabilização & 0,600 & 0,000 * & 0,269 & 0,082 & 0,314 & 0,040 * & 0,348 & 0,022 * \\
\hline Promoção da saúde & 0,475 & 0,001 * & 0,124 & 0,426 & 0,311 & 0,043 * & 0,460 & 0,002 * \\
\hline Participação comunitária e controle social & 0,230 & 0,138 & 0,027 & 0,862 & 0,162 & 0,298 & 0,403 & 0,007 * \\
\hline Vigilância à saúde & 0,302 & 0,049 * & 0,159 & 0,309 & 0,172 & 0,270 & 0,270 & 0,080 \\
\hline
\end{tabular}

Nota: para análise de correlação foram consideradas as subdimensões de atenção à saúde aos diferentes ciclos de vida, excluindo-se a subdimensão de vigilância às doenças infecto-contagiosas.

r: coeficiente de correlação de Pearson; p: nível de significância.

* Diferença estatisticamente significante.

serem assumidos por todas as equipes. Não se observou associação estatisticamente significativa entre as subdimensões de consolidação do modelo e a atenção à saúde do adolescente.

É certo que a ESF ainda enfrenta graves problemas para definir um novo modelo assistencial no país. Existem questionamentos sobre a prática cotidiana das equipes e alguns autores afirmam que as estruturas atuais ainda se mantêm permeáveis ao modelo biomédico tradicional, com reflexo negativo sobre os resultados dessas equipes 13,14 . O presente estudo corrobora essa assertiva ao registrar que maiores escores na dimensão de consolidação do modelo estão associadas a maiores escores nos resultados dos processos de trabalho das equipes.

Modelos assistenciais em saúde devem ser compreendidos como um conjunto de atividades técnicas e tecnologias que têm o objetivo de atender as necessidades de saúde de uma população. Enquanto a percepção de um novo modelo assistencial não for assumida pelas equipes da ESF, a prática centrada na consulta do médico e na abordagem da doença não será transformada. 
É preciso que os próprios integrantes das equipes assimilem a estratégia como uma prática inovadora e reestruturadora das ações de saúde, com resgate de uma visão mais ampliada do processo saúde-doença e das relações entre os membros da equipe de saúde 13,15 .

Os resultados apontam ainda algumas questões críticas que precisam ser mais bem conhecidas e discutidas no âmbito da ESF. De modo geral, existe uma percepção positiva das equipes sobre sua atuação. Outros autores já registraram que as equipes de saúde tendem a julgamentos semelhantes sobre o próprio desempenho, conforme o estudo realizado no Estado do Mato Grosso 16. De forma similar aos resultados do presente estudo, os autores registraram que médicos e enfermeiros atribuem elevados graus de aderência aos propósitos inovadores da ESF. Notifica-se, entretanto, que o instrumento utilizado referiase aos propósitos da ESF de forma genérica, enquanto a utilização do AMQ apresenta padrões bem definidos de atuação das equipes.

É importante registrar que outros autores já registraram fraco desempenho das equipes de saúde da família em relação aos eixos de "participação comunitária e controle social" e "atenção à saúde do adolescente" 16,17, assim como apontado no presente estudo a partir dos baixos escores das equipes em relação a esses itens. Sobre a participação comunitária, as equipes parecem considerá-la um atributo mais dependente da comunidade do que das suas ações. Todavia, uma análise mais criteriosa dos parâmetros do AMQ para essa subdimensão permite verificar que as ações apresentadas são pertinentes às equipes de saúde da família que, de fato, almejem a transformação do modelo assistencial. Registra-se, pois que as equipes de saúde da família ainda não compreendem a interface entre suas ações e o papel político e ideológico da participação comunitária para a consolidação do novo modelo assistencial.

Em relação à dimensão de atenção à saúde, o fraco desempenho de ações voltadas à saúde do adolescente observado no presente estudo já foi apontado em pesquisa anterior 16. As ações de atenção à saúde do adolescente ainda são recentes em todo o país e podem justificar esses resultados. Outro aspecto que pode justificar o resultado observado é que o AMQ possui apenas seis parâmetros para aferir a qualidade da assistência ao adolescente e esse dado pode representar uma limitação do próprio instrumento de coleta de dados.

Os resultados do presente estudo devem ser abordados à luz de algumas considerações sobre suas limitações. Uma delas é inerente à própria utilização do instrumento AMQ, que é um instru- mento de auto-avaliação. Alguns estudos registram que em estudos de auto-avaliação os profissionais de saúde tendem a realizar uma avaliação mais positiva de suas ações, em relação aos usuários 18,19. É possível que esse viés também exista no presente estudo, embora a entrevista realizada de forma conjunta com os profissionais de nível superior das equipes tenha buscado evitar tal situação, pois a definição de conformidade passou a ser compartilhada por três categorias profissionais (médicos, enfermeiros e cirurgiões-dentistas), limitando-se o juízo de valor pessoal nas respostas. Ressalta-se, ainda, que é importante a realização de estudos de avaliação com a participação dos atores implementadores do processo, conforme apontam Canesqui \& Spinelli 16, pois eles podem expressar adesão ou resistências em relação a nova estratégia. Na verdade a percepção de tais atores sociais pode ser bastante útil para ajustes no novo modelo. Estudo realizado no Piauí revelou que os médicos e enfermeiros da ESF não percebiam mudanças no modelo assistencial e repetiam as práticas da assistência fragmentada focada na doença 20 .

É preciso considerar ainda que os resultados do presente estudo traduzem uma particularidade do grupo de profissionais de nível superior da ESF do município avaliado. Esses profissionais apresentam um perfil diferenciado, em relação ao que se observa na maior parte do Brasil, pois mais da metade deles possui formação específica, com residência em medicina da família e comunidade ou residência multiprofissional em saúde da família. Salienta-se que a formação de recursos humanos para a ESF representa um aspecto crítico do processo de transformação do modelo assistencial 13,21.

De acordo com Gil 21, as experiências promovidas pelas residências podem representar a melhor oportunidade para uma reflexão em torno de alternativas que permitam rever os caminhos para a formação dos profissionais, na perspectiva de um trabalho mais coerente com o que se propõe através da ESF. Novos estudos são necessários para a consolidação das evidências de que o processo de formação dos recursos humanos pode interferir favoravelmente para transformação do modelo assistencial na ESF e traduzir-se em bons indicadores de saúde para a população. 


\section{Resumo}

Objetivou-se verificar a adequação de equipes de saúde da família aos padrões de qualidade do Ministério da Saúde e sua correlação com ações de atenção à saúde, segundo os profissionais de saúde. Foi utilizado o instrumento de Avaliação para Melhoria da Qualidade da Estratégia Saúde da Família (AMQ) em entrevistas conjuntas com médicos, enfermeiros e cirurgiões-dentistas. Foram definidos escores para cada uma das subdimensões avaliadas, segundo o número de conformidades aos padrões definidos no AMQ. Os escores mais baixos da dimensão de consolidação do modelo foram referentes à participação comunitária e controle social. Para a atenção à saúde, os escores mais baixos foram relativos à saúde do adolescente. As subdimensões "acolhimento e humanização" e "promoção da saúde" mostraram-se estatisticamente associados com a atenção à saúde da criança, do adulto e do idoso. A subdimensão "organização do trabalho" mostrou-se estatisticamente associada com a atenção à saúde da criança e do idoso. Existe, portanto, correlação positiva entre consolidação do modelo e melhoria da qualidade.

Qualidade da Assistência à Saúde; Pessoal de Saúde; Saúde da Família

\section{Referências}

1. Guzmán-Urrea MP. Deficiencias en los diagnósticos de las reformas sanitarias de los años noventa en América Latina. Rev Panam Salud Pública 2009; 25:84-92.

2. Conill EM. Ensaio histórico-conceitual sobre a Atenção Primária à Saúde: desafios para a organização de serviços básicos e da Estratégia Saúde da Família em centros urbanos no Brasil. Cad Saúde Pública 2008; 24 Suppl 1:S7-16.

3. Ministério da Saúde. Portaria no ${ }^{\circ}$ 648, de 28 de março de 2006. Aprova a Política Nacional de Atenção Básica estabelecendo a revisão das diretrizes e normas para a organização da Atenção Básica para o Programa Saúde da Família (PSF) e Programa Agentes Comunitários de Saúde (PACS). Diário Oficial da União 2006; 28 mar.

\section{Colaboradores}

J. M. Silva participou da elaboração do projeto, coordenação da coleta de dados, análise e interpretação dos dados e redação do texto final. A. P. Caldeira colaborou na elaboração do projeto, análise e interpretação dos resultados e redação do texto final.
4. Teixeira CF. Promoção e vigilância da saúde no contexto da regionalização da assistência à saúde no SUS. Cad Saúde Pública 2002; 18 Suppl:153-62.

5. Macinko J, Guanais FC, Souza MFM. Evaluation of the impact of the Family Health Care Program on infant mortality in Brazil, 1990-2002. J Epidemiol Community Health 2006; 60:13-9.

6. Facchini LA, Piccini RX, Tomasi E, Thumé E, Silveira DS, Siqueira FV, et al. Desempenho do PSF no Sul e no Nordeste do Brasil: avaliação institucional e epidemiológica da Atenção Básica à Saúde. Ciênc Saúde Coletiva 2006; 11:669-81.

7. Roncalli AG, Lima KC. Impacto do Programa Saúde da Família sobre indicadores de saúde da criança em municípios de grande porte da região Nordeste do Brasil. Ciênc Saúde Coletiva 2006; 11:713-24. 
8. Piccini RX, Facchini LA, Tomasi E, Thumé E, Silveira DS, et al. Necessidades de saúde comuns aos idosos: efetividade na oferta e utilização em atenção básica à saúde. Ciênc Saúde Coletiva 2006; 11:657-67.

9. Goldbaum M, Gianini RJ, Novaes HMD, César CLG. Utilização de serviços de saúde em áreas cobertas pelo Programa Saúde da Família (Qualis) no município de São Paulo. Rev Saúde Pública 2005; 39:90-9.

10. Macinko J, Almeida C, Oliveira ES, Sá PK. Organization and delivery of primary health care services in Petrópolis, Brazil. Int J Health Plann Manage 2004; 19:303-17.

11. Ministério da Saúde. Avaliação para melhoria da qualidade qualificação da Estratégia Saúde da Família. Brasília: Ministério da Saúde; 2005.

12. Venâncio SI, Silva AL, Martins MCFN, Martins PN, Figueiredo R, Rosa TEC, et al. Avaliação para a Melhoria da Qualidade da Estratégia Saúde da Família - AMQ: estudo de implantação no Estado de São Paulo. São Paulo: Instituto de Saúde; 2008.

13. Costa GD, Cotta RM, Ferreira MLS, Reis JR, Franceschini SCC. Saúde da família: desafios no processo de reorientação do modelo assistencial. Rev Bras Enferm 2009; 62:113-8.

14. Matumoto S, Fortuna CM, Mishima SM, Pereira MJB, Domingos NAM. Supervisão de equipes no Programa de Saúde da Família: reflexões acerca do desafio da produção de cuidados. Interface Comun Saúde Educ 2005; 9:9-24.
15. Senna MCM, Coehn MM. Health care model and the Family Health Strategy in the local level: analysis of an experience. Ciênc Saúde Coletiva 2002; 7:523-35.

16. Canesqui AM, Spinelli MAS. A implementação do Programa Saúde da Família em municípios do Estado de Mato Grosso, Brasil. Cad Saúde Pública 2008; 24:862-70.

17. Crevelim MA. Participação da comunidade na equipe de saúde da família: é possível estabelecer um projeto comum entre trabalhadores e usuários? Ciênc Saúde Coletiva 2005; 10:323-31.

18. Elias PE, Ferreira CW, Alves MCG, Cohn A, Kishima V, Gomes A, et al. Atenção básica em saúde: comparação entre PSF e UBS por estrato de exclusão social em São Paulo. Ciênc Saúde Coletiva 2006; 11:633-41.

19. van Stralen CJ, Belisário SA, van Stralen TBS, Lima AMD, Massote AW, Oliveira CL. Percepção dos usuários e profissionais de saúde sobre atenção básica: comparação entre unidades com e sem saúde da família na Região Centro-Oeste do Brasil. Cad Saúde Pública 2008; 24 Suppl 1:S148-58.

20. Pedrosa JIS, Teles JBM. Consenso e diferenças em equipes do Programa Saúde da Família. Rev Saúde Pública 2001; 35:303-11.

21. Gil CRR. Formação de recursos humanos em saúde da família: paradoxos e perspectivas. Cad Saúde Pública 2005; 21:490-8.

Recebido em 05/Jan/2010

Versão final reapresentada em 28/Mar/2010

Aprovado em 05/Abr/2010 